\title{
CORRECTION
}

\section{Correction to: Determining Learning Style Preferences of Learners}

\section{Sushil Shrestha* ${ }^{*}$ Manish Pokharel}

Digital Learning Research Lab, Department of Computer Science and Engineering, Kathmandu University, Nepal

Published Online: 25 January 2022

The online version of the original article can be found at https://doi.org/10.30564/jcsr.v3i1.2761

The correct Table 7 is as follows:

Table 7. Aggregation of cluster value weight of learning style ${ }^{[24]}$

\begin{tabular}{|c|c|c|c|c|c|}
\hline & & & Active & & \\
\hline & & Very weak (0) & Weak (1) & Moderate (2) & Strong (3) \\
\hline & Very weak (0) & Balanced & Moderate Active & Strong Active & Strong Active \\
\hline \multirow[t]{6}{*}{ Reflective } & Weak(-1) & Moderate Reflective & Balanced & Moderate Active & Strong Active \\
\hline & Moderate(-2) & Strong Reflective & Moderate Reflective & Balanced & Moderate Active \\
\hline & Strong(-3) & Strong Reflective & Strong Reflective & Moderate Reflective & Balanced \\
\hline & & & Visual & & \\
\hline & & Very weak (0) & Weak (1) & Moderate (2) & Strong (3) \\
\hline & Very weak (0) & Balanced & Moderate Visual & Strong Visual & Strong Visual \\
\hline \multirow[t]{3}{*}{ Verbal } & Weak(-1) & Moderate Verbal & Balanced & Moderate Visual & Strong Visual \\
\hline & Moderate(-2) & Strong Verbal & Moderate Verbal & Balanced & Moderate Visual \\
\hline & Strong(-3) & Strong Verbal & Strong Verbal & Moderate Verbal & Balanced \\
\hline
\end{tabular}

*Corresponding Author:

Sushil Shrestha,

Digital Learning Research Lab, Department of Computer Science and Engineering, Kathmandu University, Nepal;

Email: sushil@ku.edu.np

DOI: https://doi.org/10.30564/jcsr.v4i1.4400

Copyright (C) 2022 by the author(s). Published by Bilingual Publishing Co. This is an open access article under the Creative Commons Attribution-NonCommercial 4.0 International (CC BY-NC 4.0) License. (https://creativecommons.org/licenses/by-nc/4.0/). 


\section{References}

[24] Hmedna, B., El Mezouary, A., Baz, O., 2020. A predictive model for the identification of learning styles in MOOC environments. Cluster Comput. 23, 1303-

1328.

DOI: https://doi.org/10.1007/s10586-019-02992-4 